\title{
Fenomenología y psicopatología en las terapias de tercera generación.
}

\author{
Francisco Martín Murcia ${ }^{1 *}$ y Rafael Ferro García ${ }^{2}$ \\ ${ }^{1}$ Hospital Virgen del Mar, Almería, España \\ ${ }^{2}$ Centro de Psicología C.E.D.I (Granada), España
}

(Recibido 4 Febrero, 2015; Aceptado 26 Febrero, 2015)

RESUMEN: Se plantea una reconsideración de la psicopatología desde una perspectiva fenomenológica centrada en el caso de las terapias de tercera generación. Se describen los principios básicos y las bases de las actuaciones terapéuticas de la psicoterapia analítica funcional. Se presenta una forma de comprensión de la psicopatología que engloba tanto un diagnóstico transversal relacionado con la evitación disfuncional de eventos psicológicos como una teoría de la formación desadaptativa del Yo. Ambos formatos funcionales de explicación psicopatológica darían cuenta de múltiples diagnósticos clínicos al uso. Por último se concluye sobre las diferencias y dificultades conceptuales entre las distintas generaciones de terapias cognitivo-conductuales.

Palabras clave: fenomenología, psicoterapia analítica funcional, terapia de aceptación y compromiso, trastornos de personalidad.

\section{Phenomenology and psychopatology in third generation therapies}

\begin{abstract}
Reconsideration of psychopathology arises from a phenomenological perspective focused on the case of third-generation therapies. Basic principles and bases of the therapeutic actions of functional analytic psychotherapy are described. A way of understanding the psychopathology that encompasses both a transverse diagnosis related to dysfunctional avoidance of psychological events as a theory of pathological formation of Self is presented. Both functional formats of psychopathological explanation would account for multiple clinical diagnoses commonly used. Finally we conclude about the differences and conceptual difficulties between different generations of cognitive-behavioral therapies. Keywords: phenomenology, functional analytic psychotherapy, acceptance and commitment therapy, personality disorders.
\end{abstract}

\section{INTRODUCCIÓN}

Un hecho constatado en el campo de la psicoterapia es que diferentes formas de actuación (incluso de modelos psicológicos enfrentados entre sí) funcionan (APA, 2006; Hunsley, Elliot y Therrien, 2013). Para ello se ha justificado la existencia de factores comunes que serían las responsables de dichos cambios terapéuticos. Las clásicas aportaciones de Jerome Frankl (1982) y Bandura (1977) 
han sido esenciales en este terreno. Para el primero, el factor común viene dado porque el paciente padece un estado de desmoralización (incompetencia, desesperanza y desesperación) para afrontar la vida y sus problemas de forma eficaz. La terapia (sea la que sea) tiene como objetivo superar esa situación psicológica de desmoralización (incluso más que la remoción sintomática) y lo hará a través de una relación afectiva confidencial, el aumento de expectativas de solución, una explicación coherente del problema (sea un análisis funcional, cognitivo o familiar) y un conjunto de técnicas o acciones en los que ambos -paciente y terapeuta- creen. Para Bandura, el objetivo común a distintas terapias es aumentar las expectativas de auto-eficacia (es decir, la magnitud y generalidad de la autoeficiencia y la fuerza de la auto-eficacia).

También es destacable la crisis de la nosología categorial, que ha sido puesta en cuarentena cuando no apartada de la práctica clínica por sugerencia de asociaciones psiquiátricas profesionales e instituciones de salud mental (Allen, 2013).

Estos y otros aspectos contradictorios muestran que las dificultades conceptuales en Psiquiatría y Psicología no dejan de ser la espada de Damocles de estas disciplinas, aunque ha habido propuestas desde Jaspers para recolocar a la filosofía como perspectiva de resolución, entre ellas las sugeridas por Fulford, Stanghellini y Broome (2004). De hecho, se ha debatido sobre la pertinencia de acercar por ejemplo la fenomenología y el conductismo radical (Day, 1969; Fuentes y Robles, 1992). En este sentido, respecto a los enfoques clínicos, la posición de mayor ajuste a la mirada fenomenológica se da en los tratamientos denominados de tercera generación (Hayes, 2004; O’Donohue,1998; Pérez-Álvarez, 2012a).

En los años 90 aparece la tercera ola de las terapias de estirpe conductual o cognitivo-conductual, formando una amalgama de estrategias cuyos principios filosóficos están basados en una visión pragmática, determinista, idiosincrática, fenomenológica, no mentalista y holística o contextual. Algunas de ellas cuentan con su propia denominación de origen, por ejemplo la terapia de aceptación y compromiso (Hayes, Strosahl y Wilson, 1999) y la psicoterapia analítica funcional (Kohlenberg y Tsai, 1991).En ambos casos se trata de tratamientos basados en un particular punto de vista sobre la cognición y la emoción (Hayes et al, 1999;Kohlenberg y Tsai, 1994). Como se sabe, se distinguen de otros modelos clásicos de psicopatología en que no se asume necesariamente la causalidad de los eventos mentales (pensamientos, emociones o estados corporales) en el comportamiento problemático, admitiéndose explicaciones alternativas a las clásicas relaciones A-B-C (Kohlenberg y Tsai, 1991). Por tanto desde esta perspectiva no se instará a modificar al paciente y controlar dichos eventos negativos para conseguir un estado mental libre de ellos; antes bien, se procurará instruirle para aceptar dicho malestar desde una posición de seguridad (observación distanciada de la mente) y en no obcecarse con soluciones que su historia ya ha descrito como ineficaces. La teoría de la aceptación (Hayes et al., 1999) se basa precisamente en el análisis de los costos personales que tiene para el paciente dicha servidumbre evitativa, aun conviniendo con el paciente los beneficios secundarios de su forma de vivir. El análisis de los valores entendidos como direcciones 
vitales sería una de las tareas esenciales. Por su parte, el formato terapéutico de Kohlenberg y Tsai $(1991,1995 a)$ se basa en la implementación de ciertos principios derivados del conductismo radical en los problemas cotidianos que tienen muchos pacientes de larga evolución (enmarcados en los tradicionales trastornos de la personalidad), como las dificultades en las relaciones íntimas, aislamiento, alexitimia, baja tolerancia a la crítica o al fracaso, hostilidad, fobia social o conductas compulsivas (Kohlenberg y Tsai, 1991). Se focaliza esencialmente en las contingencias que ocurren in vivo en la sesión, entendiendo que existe una equivalencia funcional entre el contexto terapéutico y la vida del cliente. Se atiende a las conductas problemáticas y a los cambios positivos, procurando fortalecer repertorios de conducta más adaptativos a través del reforzamiento natural y el moldeamiento en sesión. La observación de que el mero hecho de atender a las conductas que forman parte de la clásica Transferencia facilita las mejorías, hace que desde este enfoque se sugiera un esfuerzo en el uso funcional de la misma. Las claves operativas de este enfoque incluyen buscar conductas problema, evocarlas en sesión, facilitar y reforzar conductas de cambio positivo, observar los efectos reforzantes del terapeuta (que lógicamente variarán según el problema, los objetivos y las personas) y adiestrar al paciente a entender funcionalmente su comportamiento problemático.

Estos formatos terapéuticos tienen como premisa común la des-psicologización de los problemas psicológicos y así acceder a las psicologías efectivas (Fuentes, 1994). Por un lado se le permite al paciente que experimente los límites y los enredos de la vía psicológica para la verdadera re-estructuración personal (y de paso se defienda con éxito de las modas new-age para la supuesta resolución de los problemas psicológicos). Por otra, se reforzará su responsabilidad éticomoral para hacerse cargo de una relación terapéutica compleja que no servirá para "que se sienta bien" sin más, sino para que aprenda trabajando aunque duela.

\section{PSICOPATOLOGÍA}

Desde la terapia de aceptación y compromiso, se ha descrito un trastorno transversal o común a distintas patologías basado en la evitación experiencial o evitación sistemática y desadaptativa de eventos mentales aversivos. Se trata de un patrón inflexible que consiste en vivir al servicio de eludir cualquier forma de malestar psicológico en forma de pensamientos, sensaciones corporales, recuerdos y otro tipo de fenómenos privados (síntomas habituales en trastornos psicopatológicos de cualquier tipo). Para ello, la persona genera un amplio repertorio de respuestas con esa función y acaba sumido en la paradoja expuesta por Watzlawick de que la "solución es el problema" (Watzlawick, Weakland y Fish, 1976). Aunque evitar el malestar es en muchas ocasiones adaptativo, se observa que las personas que presentan psicopatología precisamente la tendrán por la propia inflexibilidad de su patrón de conducta, de tal forma que el sentido de su vida gira en torno a sus síntomas y la necesidad de evadirse de ellos. Aunque de forma inmediata suelen encontrar alivio (véanse por ejemplo las compulsiones, 
los rituales mentales, el uso de alcohol y drogas, la toma de psicofármacos, no salir de las zonas de seguridad agorafóbica) lo cierto es que o bien aparecen los síntomas con más virulencia o bien se desplazan en el sentido freudiano.

El trastorno por evitación experiencial es un diagnóstico exclusivamente funcional, es decir establece las relaciones contingenciales que resultan en un problema psicológico para el paciente (Hayes et al, 1999).

Por otra parte, el modelo explicativo de la formación del Yo de Kohlenberg y Tsai (1991, 1995b) permite explicar los clásicos trastornos de personalidad. Por de pronto, el desarrollo desadaptativo del Yo se daría en el contexto de crianza del niño y el grado de severidad en el problema dependerá de la falta de control privado sobre las unidades funcionales "Yo X" que los padres no habrían enseñado correctamente. La clave en el desarrollo del Yo radica en transferir el control de estas respuestas de lo público a lo privado. La cantidad de experiencias privadas relacionadas con el Yo nos podrían indicar la normalidad de esta experiencia. Así, el grado de dificultad experimentado por un individuo puede variar en función del grado de control privado sobre sus respuestas "Yo X". En general, cuantas menos respuestas de este tipo tenga una persona bajo control privado, mayor será la confusión o dificultad al contestar preguntas que tengan que ver con sus preferencias personales, sus deseos o sus valores y, en general, con lo que tenga que ver con él mismo. La cuestión no es que se haya suprimido el reporte verbal de sentimientos o necesidades sino que no se han determinado con precisión los estímulos antecedentes de nuestras experiencias corporales (estímulos privados como sensaciones o sentimientos) o necesidades (reforzadores). Dependiendo del tipo de entrenamiento recibido puede que estén alteradas algunas o todas las relaciones "Yo X" (sentimientos, opiniones, actitudes, necesidades, etc.) y pueden estar influidas en función de quien esté presente es decir, si estamos en compañía de alguien (íntimo o casual) o solos. Esta información puede ser crucial en el tratamiento.

Desgraciadamente, es usual que algunos miembros del ambiente socio-familiar invaliden la experiencia privada del niño. Se ilustrará con algunos ejemplos: cuando se castigan verbalizaciones como "no tengo más hambre", diciéndoles "es imposible porque no has comido nada"; o estableciendo mal las relaciones funcionales, "estoy nervioso, me duele la barriga", diciéndoles: "no estás nervioso, tú lo que tienes es hambre"; castigando la espontaneidad y la creatividad "he visto un pez naranja", "anda ya, no digas tonterías"; suplantando los deseos del niño, "tu quieres comer, dormir, esto no te gusta, aquello si"; reforzando los deseos y necesidades del niño en función de los propios deseos o estado de ánimo del cuidador (dado por ejemplo en algunos casos cuya madre presenta un trastorno de personalidad límite).

Las personas con trastornos leves/moderados del Yo pueden tener un número elevado de respuestas "Yo-X" evocadas por estímulos privados pero también por estímulos bajo control público o externo. Así su sentido del Yo se verá afectado por la presencia o ausencia de otras personas y de sus opiniones y estados emocionales, como se ha mencionado. Los autores aclaran que no es un problema 
de habilidades sociales, es decir que sepan lo que quieran y no sean capaces de expresarlo, sino que el problema es que lo que se siente o desea está bajo control de los demás. Esto dará lugar a experiencias de un "sí mismo inseguro y/o inestable" en personas cuyas opiniones, necesidades, etc. dependen de quién esté presente. Ejemplos habituales en la clínica serían aquellas personas que cambian radicalmente en situaciones de intimidad respecto a las relaciones formales, que "dejan de ser ellas" cuando tienen pareja, que pueden confundir lo que quieren con lo que quieren los demás o que necesitan un estímulo público para "querer" algo. Otra situación paradigmática es la presencia de una sensibilidad extrema a las opiniones y críticas de los demás cuando se tiene un sentido del Yo inestable y controlado públicamente, pues habitualmente se sentirá vulnerable y emergerán respuestas defensivas ante la más mínima crítica. La extremada sensibilidad a las opiniones, creencias, deseos y humor de los demás es otra forma de decir que los eventos "yo X" de una persona, que deberían estar bajo control privado están realmente bajo control público. Este entrenamiento va a suponer que unidades como "Yo pienso..", "Voy a hacer.." están bajo control externo y por tanto presentan una fuerte dependencia en los demás. Además, en los casos donde no se ha generado correctamente la perspectiva, supone una experiencia del Yo como vacía, caótica, impredecible o falta del sentido. Hay otras patologías del yo, como el trastorno narcisista de la personalidad y el trastorno de personalidad límite en los cuales la emergencia del sentido del Yo puede ser explicado desde esta formulación. Es importante resaltar que los trastornos leves/moderados y los graves no son excluyentes, como bien se observa en la práctica clínica.

Esta teoría de la formación del Yo propone también formas de intervención para intentar resolver esa falta de control privado de la experiencia de Ipseidad (Kohlenberg y Tsai, 1991; Kohlenberg, Tsai, Kanter y Parker, 2009). Quizás, la recomendación más general sea el reforzamiento de cualquier expresión en ausencia de claves o indicaciones externas específicas. Como se ha descrito antes, muchas de sus conductas están bajo control de estímulo de otros y no de sus estímulos privados (sensaciones, necesidades, etc.). El trabajo del terapeuta sería poner bajo el control privado lo que dice o hace el paciente. Para ello habría que estimular y reforzar la conducta verbal del cliente en ausencia de indicaciones externas específicas. En otras palabras, la intervención consiste en fortalecer todas las unidades "Yo X" bajo control privado, lo cual ayudará también a la emergencia final del control privado sobre el "Yo". Tareas sin claves externas pueden ser los tests proyectivos o asociaciones libres de ideas, sin necesariamente el propósito de descubrir significados ocultos sino el mero hacer-decir. Para esto hay que ajustar las tareas terapéuticas al nivel de control privado del repertorio del cliente. Es muy importante estar atento a ello, ya que si el ajuste no es adecuado, el paciente podría tener una experiencia aversiva y aparecerán con probabilidad conductas de evitación. Los autores proponen modificar estas tareas en cuatro aspectos. Primero, se presentan al paciente tareas cuya importancia radica en el proceso de evocar estas unidades "Yo X" bajo control privado. Segundo, adaptarlas para que el grado de control privado requerido sea adecuado 
al repertorio del cliente. Tercero, reforzar el máximo de unidades "Yo X" como sea posible. Finalmente, el terapeuta debe ser sensible a la aparición de otras conductas clínicas no relacionadas con los trastornos del yo que sean oportunidades terapéuticas.

En el reciente manual (Kohlenberg, Tsai, Kanter y Parker, 2009) se proponen además, unas nuevas implicaciones clínicas y técnicas para promocionar el mindfulness, en especial la observación o tomar conciencia de lo que está sintiendo. Para esto, se sugieren ejercicios experienciales similares a los propuestos por la terapia de aceptación y compromiso (ACT) que tienen su base en la respiración y en la observación no crítica o valorativa de pensamientos y emociones, focalizándose en el aquí y el ahora.

Las implicaciones clínicas del desarrollo de la perspectiva del yo siguen el modelo de intervención de ACT. Las tres definiciones del Yo que propone ACT están relacionadas con los marcos relacionales deícticos (Foody, Barnes-Holmes y Barnes-Holmes, 2012). Los tres sentidos del Yo para ACT son: el Yo como contenido (o mejor, el contenido del yo), el Yo como proceso y el Yo como contexto. Podría traerse a colación en este sentido los tres niveles del yo propios de la investigación fenomenológica; el yo pre-reflexivo o básico (la conciencia implícita de lo que es mi experiencia en primera persona), el yo reflexivo (la conciencia de un si mismo como invariante y persistente) y el nivel narrativo del yo, en el que se da cuenta de uno mismo en términos históricos o autobiográficos (Pérez-Álvarez, 2012b).

En definitiva, una parsimoniosa manera de entender como se gestan los problemas clínicos que no implique dificultades objetivas de razonamiento y unos formatos de intervención fenomenológica (sobre lo que ocurre en sesión), podrían ser una buena alternativa a la situación de sobresaturación psicológica.

\section{CONCLUSIONES}

Dado que las dificultades conceptuales en los distintos paradigmas se observan tanto a nivel ontológico (sobre qué es el ser humano y qué son los trastornos psicológicos) como epistemológico (qué vía puede tomarse para acceder al conocimiento) y pragmático (en fin, el mundo de las terapias), se hace necesario un repaso de las cuestiones filosóficas de base para ver el sentido de las terapias de tercera generación frente a otros paradigmas.

Las psicologías representacionales han dado en su vertiente aplicada las denominadas primera y segunda generación de terapias cognitivo-conductuales. Escoger este planteamiento representacional cartesiano (psicología cognitiva, neurociencia) llevaría a una dificultad objetiva de razonamiento (Robles, 1996), por la que el punto de partida ya en si lastra la posibilidad de resolución. Esto ya estaría denunciado por Jaspers (1993) como prejuicio representativo. Y aunque ello no sea óbice para que las intervenciones terapéuticas funcionen (se recordarán de nuevo los factores comunes), es importante observarlo. A pesar de la bondad objetivista del planteamiento heredero de Descartes, esto es, la mensuración 
necesaria de la confusa y subjetiva experiencia fenoménica, lo cierto es que a partir de esta pretensión se produce el alejamiento del mundo real y circundante en el que la persona está, para dotar de relevancia al ámbito de las ideas, al mundo re-presentado. Esta duplicación en la que la conciencia estaría frente a una presencia "idealizada" o subjetiva proporcionada como el mundo exterior no es necesaria para la comprensión del hecho psicológico.

La fenomenología podría ser una vía que diera semejantes condiciones de posibilidad, dado que es un método de constatación y descripción de la experiencia (del fenómeno en sí). La explicación del hecho psicológico, si se encontrara, siempre estaría en el mismo orden de realidad (por ejemplo, en la constitución indisoluble de la triple relación contingencial conducta-situación-reforzador) y no en otro orden (sean complejos de Edipo o déficits de serotonina). Fenomenología y conductismo radical compartirían el objetivo de explicar el hecho psicológico en el mismo orden de realidad, sin negar el mundo ahí dado y sin caer en el subjetivismo en el que el mundo pareciera ser una mera autoexpresión del acto cognoscitivo del sujeto (Frankl, 1967). De entrada, la pretensión de la fenomenología es no reificar las experiencias psicológicas (Catania, 1998); por ejemplo la sustancialización de la conciencia no ofrece garantías dado que no se experimenta la conciencia, sino que se es consciente de algo. La conciencia es siempre intencional y esta intencionalidad es una manera de referirse a variables que controlan el comportamiento (Skinner,1953; Robles, 1996b). Específicamente la conciencia de si-mismo podría estar restringida a la capacidad de tener lenguaje y a las prácticas sociales que nos han hecho "caer en la cuenta" de distintas experiencias (sensaciones, percepciones y, en definitiva, todo tipo de eventos privados). Y la historia vivida de cada individuo sería un modelado continuo, o en palabras de Jaspers "el hombre como posibilidad abierta, como ser no terminado, es decir, que siempre es más y otra cosa que lo que ha realizado de si, pues está abierto a la experiencia del mundo" (Jaspers, 1993).

En resumen, es la historia ambiental y conductual idiosincrática la que influye en la experiencia de recordar, pensar o sentir en el presente. Es decir, el mundo en el que las personas viven y se desarrollan. Y ese mundo estaría antes que las ideas que sobre él se desprendan. Las creencias, los valores, serían el horizonte de sentido hacia el que se mueve el ser humano y estarían insertadas en su ser como ser psicológico, no se trataría de un proceso de razonamiento ni se accede a ellos analíticamente dado que están ahí (se quiera o no).

Pues bien, el método fenomenológico propio del conductismo radical salvaría los prejuicios y el confuso contubernio denunciado por Ortega en la lección III de sus Investigaciones Psicológicas (Ortega, 1979) al retornar a las cosas mismas sorteando entre otros, el sesgo de la parcelación científica y la reificación (algo que reina en psicopatología). 


\section{DISCUSIÓN}

La propuesta de aprehender la psicología para evitar su encalle en propuestas aporéticas representacionales, permite llegar a una elegante manera de entender la naturaleza psíquica del ser humano, de sus trastornos y de sus tratamientos. La mirada fenomenológica salvaría esas barreras intelectuales y teóricas y de paso podría salvar a la psicología y re-colocarla por derecho al lado (por no decir enfrente) de posturas biologicistas, mecanicistas y cerebro-centristas (PérezÁlvarez, 2011).

Puede desprenderse del presente trabajo que las terapias de tercera generación se ajustan a una visión pragmática, determinista, fenomenológica, idiosincrática, no mentalista y holística o contextual. De igual manera, destaca la profundidad de comprensión y análisis del comportamiento humano (incluyendo los aspectos más complejos del psiquismo) que están en la base experimental y aplicada de estas terapias. En particular y como propone Pérez-Álvarez (2012b), la psicoterapia analítica funcional establece una relación terapéutica fundada en una actitud fenomenológica que proporciona un contexto donde el paciente puede desarrollar una perspectiva de primera persona y una autoconciencia prereflexiva más robusta, que permitiría un tratamiento adecuado de los clásicos trastornos de la personalidad y de las alteraciones del Yo. Por otra parte, la descripción de un trastorno transversal exclusivamente funcional es muy interesante por su parsimonia y por la posibilidad de actuar sobre las conductas problema con conocimiento de causa.

Quedarían por describir de forma elaborada los fenómenos esenciales de solipsismo-reflexividad y quasi-solipsismo (experiencia del cuerpo fenoménico y la inflexibilidad cognitiva) y la preocupación por "ser", dado que entendemos que podrían aportar una base transversal para una comprensión cabal de los trastornos psicopatológicos, insertada en una psicología de alcance.

\section{REFERENCIAS}

APA Task Force on Evidence-Based Practice. (2006). Evidence-based practice in psychology. American Psychologist, 61, 271-285.

Allen, F. (2013). Saving normal: Aninsider's revolt against out-of-control psychiatric diagnosis, DSM-5, bigpharma, and the medicalization of ordinary Life. New York: Harper Collins.

Bandura, A. (1977). Self- Efficacy: Toward a unifying theory of behavior change. Psychological Review, 84, 191-215.

Catania, A. C. (1998). Learning (4th ed.). UpperSaddleRiver, NJ: Prentice Hall.

Day, W. (1969). Radical behaviorism in reconciliation with phenomenology. Journal of The Experimental Analysis of Behavior, 12, 315-328.

Foody, M., Barnes-Holmes and Barnes-Holmes, D. (2012). The Role of Self in Acceptance and Commitment Therapy. En L. McHugh y I. Stewart (Eds.), The Self and perspective taking. Contribution and applications from 
modern behavioral science. (pp. 125-159). Reno: Context Press

Frank, J. (1982). Therapeutic Components Shared by all Psychotherapies. En J. Harvey, M. Parks (Eds.), Psychotherapy Research and Behavior Change. Washington: APA.

Frankl, V. (1967). Psychotherapy and Existentialism. Selected Papers on Logotherapy. London: SouvenirPress.

Fuentes, J.B. y Robles, F.J. (1992).The Phenomenological Nature of Skinner's Radical Behaviourism. Revista de Historia de la Psicología, 2, 61-67.

Fuentes, J.B. (1994). Introducción del concepto de "conflicto de normas irresuelto personalmente" como figura antropológicas (específica) del campo psicológico. Psicothema, 6, 3, 421-446.

Fulford, K, Stanghellini, G. y Broome, M. (2004). What can philosophy do forpsychiatry? WorldPsychiatry, 3, 130-135.

Hayes, S.C., Strosahl, K.D., Wilson, K.G. (1999).Acceptance and Commitment Therapy. New York: Guilford Press.

Hayes, S. C. (2004). Acceptance and commitment therapy, relational frame theory, and third wave of behavioral and cognitive therapies. Behavior Therapy, $35,639-665$.

Hunsley, J., Elliot, K. y Therrien, Z. (2013). The efficacy and efectiveness of psychological treatments. The Canadian Psychology Association Press.

Kohlenberg, R.J. y Tsai, M. (1991). Functional analytic psychotherapy.Creating intense and curative therapeutic relationship. New York: PlenumPress.

Kohlenberg, R.J. y Tsai, M. (1994) Functional Analytic Psychotherapy: A Radical Behavioral Approach to Treatment and Integration. Journal of Psychotherapy Integration, 4, 174-201.

Kohlenberg, R.J. y Tsai, M. (1995a).Functional analytic psychotherapy: a behavioral approach to intensive treatment. En W. O'Donohue y L. Krasner (Eds.), Theories of behavior therapy. Exploring behavior change, (pp. 637658). Washington: APA.

Kohlenberg, R.J. y Tsai, M. (1995b). I speak, therefore I am: A behavioral Approach to understanding problems of the self. TheBehaviorTherapist, 18, 113-124.

Kohlenberg, R. J., Tsai, M. y Kanter, J. W. (2009). What is Functional Analytic Psychotherapy? En M. Tsai, R. J. Kohlenberg, J. W. Kanter, B. Kohlenberg, W.C. Follette, y G. M. Callaghan, G.M. (Eds). A Guide to Functional Analytic Psychotherapy Awareness, Courage, Love and Behaviorism (pp. 1-19). New York: Springer.

Kohlenberg, R.J., Tsai, M., Kanter, J. W. y Parker, C.R. (2009). Self and Mindfulness.In Tsai, M., Kohlenberg, R.J., Kanter, J.W., Kohlenberg, B., Follette, W. y Callaghan, G.M. (Eds.). A Guide to Functional Analytic Psychotherapy. Awareness, Courage, Love, and Behaviorism (pp. 103-130). New York: Springer.

Jaspers, K. (1993, orig. 1913). Psicopatología General. México: Fondo de Cultura Económica. 
O'Donohue, W. (1998). Conditioning and third-generation behavior therapy. En W. O'Donohue (Ed.). Learning and behavior therapy (pp. 1-14). Needham Heights: Allyn and Bacon.

Ortega y Gasset, J. (1979). Investigaciones psicológicas, en Obras Completas. Madrid: Alianza Editorial.

Pérez-Álvarez, M. (2011). El mito del cerebro creador. Cuerpo, conducta y cultura. Madrid: Alianza Editorial.

Pérez-Álvarez, M. (2012a). Third-Generation Therapies: Achievements and challenges, International Journal of Clinical and HealthPsychology, 12 (2), 291310.

Pérez-Álvarez, M. (2012b). Las raíces de la psicopatología moderna. La melancolía y la esquizofrenia. Madrid: Ed. Pirámide.

Robles, F.J. (1996a). Para aprehender la psicología. Un análisis histórico-epistemológico del campo psicológico. Madrid: Siglo XXI.

Robles, F.J. (1996b). Naturaleza y sentido del campopsicológico a la luz del concepto de inminencia operatoria, Psicothema, 8, 613-623.

Skinner, B.F. (1953). Science and human behavior. New York: Macmillan. Watzlawick,P. Weakland, J.y Fish, R. (1976). Cambio: formación y solución de los problemas humanos. Barcelona: Herder. 